\title{
Update on Treatment and Resistance of Human Trichuriasis
}

\author{
Ayola Akim Adegnika ${ }^{1,2,3,4} \cdot$ Felix Lötsch ${ }^{1,5} \cdot$ Regis Maurin Obiang Mba ${ }^{1} \cdot$ \\ Michael Ramharter ${ }^{1,2,5}$
}

Published online: 28 September 2015

(C) Springer International Publishing AG 2015

\begin{abstract}
Trichuris trichiura is a common soil-transmitted helminthic pathogen with considerable impact on human health. To achieve appropriate control of trichuriasis from a public health perspective, effective treatments and regular education of populations most at risk such as children and pregnant women are essential. Currently available drugs show however only unsatisfying cure rates when used in shortcourse regimens, and egg reduction rates are disappointingly low. An improvement in efficacy of drug therapy has been demonstrated for a prolonged 3-day dosing regimen of albendazole as well as for a combination therapy of albendazole and oxantel pamoate or mebendazole and albendazole. However, even these regimens do not reach the widely accepted threshold for a satisfactory cure rate of at least $90 \%$. While this lack of efficacy of current anthelminthic drugs may be explained by specific single nucleotide polymorphisms in the $\beta$-tubulin gene of T. trichiura, these findings
\end{abstract}

This article is part of the Topical Collection on Topics Exploring Loa-Loa, Onchocerciasis, Hookworm, Ascaris, Trichuris

Ayola Akim Adegnika
aadegnika@gmail.com

1 Centre de Recherches Médicales de Lambaréné (CERMEL), Hôpital Albert Schweitzer, BP: 118, Lambaréné, Gabon

2 Institute of Tropical Medicine, University of Tübingen, Tübingen, Germany

3 Department of Parasitology, Leiden Medical University Center, Leiden, The Netherlands

4 African Partner Site, German Centre for Infection Research (DZIF), Lambaréné, Gabon

5 Department of Medicine I, Division of Infectious Diseases and Tropical Medicine, Medical University of Vienna, Vienna, Austria highlight the need for further research to develop highly efficacious short-course treatments for human trichuriasis.

Keywords Trichuriasis $\cdot$ Treatment $\cdot$ Albendazole . Mebendazole $\cdot$ Resistance $\cdot \beta$-tubulin

\section{Introduction}

Trichuris trichiura, or whipworm, is a soil-transmitted helminth (STH) - a group consisting of pathogens including $A s$ caris lumbricoides, Strongyloides stercoralis, Necator americanus and Ancylostoma duodenale. STH occurs almost globally with higher prevalence in developing countries and resource-limited regions in the tropics such as parts of subSaharan Africa, East Asia, China, India and South America [1-3]. Infection rates typically peak in school children [4-7] and, to a lesser extent, in pre-school children [7]. Importantly, pregnant women constitute another population with increased prevalence and morbidity [8]. Behavioural factors and lack of infrastructure such as safe and reliable water supply and appropriate sanitation in school and at home are the major risk factors for infection $[6,9]$. Thus, school children in highly endemic regions represent the target population for appropriate control measures, such as mass drug administration and mass education. Approximately 800 million people are infected with $T$. trichiura globally $[10,11 \bullet]$, and the number of disability-adjusted life years (DALYs) is estimated to range between 40 and 60 million $[12,13]$. However, there is controversy with regard to the global burden of STH as most infections are asymptomatic and therefore often overlooked [9]. Lightly infected populations appear generally healthy and will only be aware of their infection status during a routine checkup for STH. However, in settings characterized by coinfections with other pathogens, nutrient deficiencies or in 
cases of chronic and high-burden infections with STH, anaemia may be observed in pregnant women [8, 14, 15], and stunting, physical and cognitive retardation and anaemia in school children [16]. Moreover, STH can cause malabsorption leading to impaired digestion and poor absorption of nutrients by alterations of the mucosa of the gastrointestinal tract [17]. Furthermore, an association between STH infection during pregnancy and poor cognitive and gross motor outcomes [18] as well as poor vaccine immune responses [19] in both unborn infants and school-aged children [20-22] has been reported. Thus, STH is considered as an important public health problem in affected tropical regions.

To address STH infection control, the 24th World Health Assembly passed in 2001 a resolution (WHA. 45.19) [23, 24] affirming that the control of schistosomiasis and soiltransmitted helminthiases should be considered as a public health priority [23] and urging the member states to put in place measures including large-scale use of anthelminthic drugs for school-aged children in less developed countries [24]. In daily practice in resource-limited settings, presumptive treatment is therefore commonly administered systematically to target populations without prior examination of stool samples. Such treatments are usually either albendazole (400 mg) or mebendazole (500 mg) single-dose therapy or 3 days twice daily mebendazole $(100 \mathrm{mg})$. Whereas these regimens have satisfactory efficacy against other STH, cure rates for trichuriasis are notoriously poor and rarely exceed $50 \%$ [25•, 26•, 27, 28•, 29•, 30•]. This review summarizes recently published data advancing our knowledge of anthelminthic drug therapy for human trichuriasis.

\section{Methodology}

Medline and Google Scholar were searched for relevant publications of clinical trial data assessing established anthelminthic drugs for human trichuriasis. Publications evaluating potential genetic targets associated with resistance of STH were also identified for this review. Data published between 2010 to mid-2015 are reviewed here.

\section{Results}

\section{Clinical Trials of Established Anthelmintics Against T. trichiura}

The most widely used treatment for $T$. trichiura consists of a single dose of $400 \mathrm{mg}$ of albendazole, despite the fact that this regimen has rarely been systematically evaluated. Albendazole is furthermore currently considered as the drug of choice for repeated mass drug administrations in endemic areas. The choice of albendazole is based on its cost- effectiveness, safety and logistical advantages as a singledose treatment [25•]. The aim of mass treatment is generally not a complete cure from helminthic infections but rather a significant reduction of worm burden and consecutively egg excretion - potentially leading to a reduction of morbidity and transmission within the community [31]. The target population of such mass treatments remains school children [23, 24, 25•]. For pregnant women, the World Health Organization recommended in 1994 to treat all pregnant women against hookworms due to the concern of hookworm-induced anaemia [32] in highly endemic areas. Besides albendazole, other anthelminthic drugs and drug combinations are also used (e.g. mebendazole or oxantel pamoate) $[26 \bullet, 33 \bullet]$ or are under evaluation (nitazoxanide) [29•].

\section{Albendazole}

Albendazole is a broad-spectrum anthelminthic drug with good efficacy against most soil-transmitted helminths. Chemically, it is a methyl 5-(propylthio)-2-benzimidazolecarbamate $\left(\mathrm{C}_{12} \mathrm{H}_{15} \mathrm{~N}_{3} \mathrm{O}_{2} \mathrm{~S}\right)$. Albendazole is used as a single dose (400 mg) in mass drug administration campaigns. Several studies reported its good effectiveness and positive effects in school children such as an increase in haemoglobin levels, improvement of appetite, fitness and cognitive performance [34-36].

In the general population, albendazole is usually well tolerated and the only formal contraindication besides pregnancy is allergy to imidazole derivatives. Some controversy exists in the classification of albendazole for its use during pregnancy. It has been shown to have teratogenic and embryotoxic effects in rats and rabbits. Albendazole must therefore not be administered during the first trimester [10, 37]. Since it is not known whether albendazole has harmful effects on the unborn human child during the second and third trimester of pregnancy, it is considered as a category $\mathrm{C}$ substance by the Food and Drug Administration (FDA). The clinical benefit of albendazole in pregnant women must therefore outweigh the potential risks of this drug. However, recent trials in Uganda [37-40], Sri Lanka [41, 42], and Sierra Leone [43, 44] indicate the safety of its use after the second trimester of pregnancy.

Efficacy of albendazole is rather low against T. trichiura in comparison to other STH. In clinical trials, cure rates (CR) of single-dose albendazole for T. trichiura ranged between $2.6 \%$ [0.0-5.6\%] [26•] and 64.5\% [44.4-84.7\%] [45•], and egg reduction rates (ERR) were between $7 \%[-52-78 \%][25 \bullet]$ and $83.1 \%$ [78.5-91.4\%] [28•] (see Table 1). Two doses of albendazole given on two consecutive days resulted in improved CR of $67 \%$ [52-82\%] [25•] and up to $83 \%$ [73$93 \%$ ] [25•] when given on three consecutive days. Doubling the dose of albendazole even when administered as a single dose also led to higher CR (43.4\% [32.2-54.6\%]) [46•] compared to the standard dose (15.4\% [8.4-20.6\%]). Thus, 
Table 1 Summary of cure rates and egg reduction rate of monotherapies in trichuriasis

\begin{tabular}{|c|c|c|c|}
\hline Antihelminthic & Regimens & $\begin{array}{l}\text { Cure rate in \% } \\
{[95 \% \mathrm{CI}]}\end{array}$ & $\begin{array}{l}\text { Egg reduction } \\
\text { rate in } \%[95 \% \mathrm{CI}]\end{array}$ \\
\hline \multirow[t]{12}{*}{ Albendazole } & \multirow[t]{7}{*}{1 dose } & $40[26-54][25 \bullet]$ & $7[-52-78][25 \bullet]$ \\
\hline & & $64.5[44.4-84.7][45 \bullet]$ & Not available \\
\hline & & $\begin{array}{l}30.8[23,24,25 \bullet, 26 \bullet \\
\quad 27,28 \bullet, 29 \bullet, 30 \bullet, 31 \\
32,33 \bullet, 34-39][28 \bullet]\end{array}$ & 83.1 [78.5-91.4] [28•]; \\
\hline & & $33.8[22.6-46.6][47 \bullet] ;$ & $76.7[62.6-86.1][47 \bullet]$ \\
\hline & & $2.6[0.0-5.6][26 \bullet] ;$ & $45.0[32.0-56.4][26 \bullet]$ \\
\hline & & $15.4[8.0-22.8][46 \bullet] ;$ & $54.9\left[46{ }^{\bullet}\right]^{\mathrm{d}}$ \\
\hline & & $14.5[8.4-20.6][46 \bullet]$ & $45.6[25.9-61.0][46 \bullet]$ \\
\hline & 2 doses & $43.4[32.2-54.6]^{\mathrm{a}}[46 \bullet]$ & $89.3^{\mathrm{a}}[46 \cdot]^{\mathrm{d}}$ \\
\hline & 2 doses & $67[52-82]^{\mathrm{b}}[25 \bullet]$ & $58[29-100]^{\mathrm{b}}[25 \bullet]$ \\
\hline & \multirow[t]{3}{*}{3 doses } & $83[73-93]^{\mathrm{c}}[25 \bullet]$ & $91[83-100]^{\mathrm{c}}[25 \bullet]$ \\
\hline & & $19.6[12.0-29.1]^{\mathrm{c}}[30 \bullet]$ & $88.8[80.9-94.7]^{\mathrm{c}}[30 \bullet]$ \\
\hline & & $56.2[41.2-70.5]^{\mathrm{c}}[47 \bullet]$ & $94.0[89.4-96.8]^{\mathrm{c}}[47 \bullet]$ \\
\hline \multirow[t]{7}{*}{ Mebendazole } & \multirow[t]{5}{*}{1 dose } & $8.4[3.1-13.8][33 \bullet] ;$ & $58.5[45.2-70.9][33 \bullet] ;$ \\
\hline & & $63.1[51.6-74.6][45 \bullet]$ & not available \\
\hline & & $39.7[27.6-52.8][47 \bullet] ;$ & $82.5[71.0-89.6][47 \bullet]$ \\
\hline & & $11.8[5.7-17.9][26 \bullet] ;$ & 75.0 [64.2-82.0] [26•]; \\
\hline & & $20.4[12.4-28.4][46 \bullet]$ & $66.7\left[46^{\bullet}\right]^{\mathrm{d}}$ \\
\hline & 2 doses & $41.9[31.9-52.0]^{\mathrm{a}}[46 \bullet]$ & $94.3^{\mathrm{a}}[46 \cdot]^{\mathrm{d}}$ \\
\hline & 3 doses & $70.7[57.3-81.9]^{\mathrm{c}}[47 \bullet]$ & $97.3[94.9-98.8]^{\mathrm{c}}[47 \bullet]$ \\
\hline Oxantel pamoate & 1 dose & $26.3[18.1-34.5][26 \bullet]$ & $93.2[90.0-95.7][26 \bullet]$ \\
\hline Nitazoxanide & 1 dose & $6.6[2.4-10.8][29 \bullet]$ & $13.4[0.0-33.7][29 \bullet]$ \\
\hline
\end{tabular}

${ }^{a}$ Drugs were given $8 \mathrm{~h}$ apart on the same day

${ }^{\mathrm{b}}$ Drugs were given on two consecutive days

${ }^{\mathrm{c}}$ Drugs were given on three consecutive days

d $95 \%$ confidence intervals are missing in this study for ERR efficacy of albendazole is dependent on the dose and the length of the regimen. This is supported by the fact that the ERR was lowest when given as a single dose $7 \%$ [-52-78 \%] [25.] and highest in a regimen of three consecutive doses $(94.0 \%$ [89.4-96.8\%]) [47•].

\section{Mebendazole}

Mebendazole is a broad-spectrum anthelmintic drug with satisfactory efficacy against most intestinal nematodes with the exception of Trichuris spp. Toxicity is low and the drug is usually very well tolerated. Just as albendazole, it is classified as a pregnancy class $\mathrm{C}$ drug by the FDA and it has no other formal contraindication other than allergy to imidazole derivatives. Recommended dosing regimens commonly require 3 days twice daily dosing rendering this drug less attractive for mass treatment campaigns compared to albendazole. A single dose of $500 \mathrm{mg}$ is used as well but less efficacious.

Recent clinical trials confirmed the low efficacy of mebendazole against Trichuris spp. A one dose $500 \mathrm{mg}$ therapy showed a CR of $8.4 \%$ [3.1-13.8\%] in one trial [33•] and a relatively high $\mathrm{CR}$ of $63.1 \%$ [51.6-74.6\%] in another one [45•]. Highest CR and ERR were achieved in a trial with three doses (cure rate of $70.7 \%$ [57.3-81.9\%] and ERR of $97.3 \%$ [94.9-98.8\%], respectively [47•]) (Table 1).

\section{Other Anthelminthic Drugs Against T. Trichiura}

Oxantel pamoate and nitazoxanide - currently evaluated for the treatment against $T$. trichiura-have shown only poor CR so far (26.3\% [18.1-34.5\%] [26•] and $6.6 \%$ [2.4$10.8 \%$, respectively). However, the ERR of oxantel pamoate was satisfactory at $93.2 \%$ [90.0-95.7\%] [26•], compared to that of nitazoxanide at $13.4 \%[0.0-33.7 \%]$ [29•].

\section{Trials Evaluating Combination Therapy}

Several regimens with a combination of drugs have been assessed over the past years [26•, 29•, 33•, 46•] (Table 2). The combination yielding the highest efficacy for Trichuris spp. was albendazole plus oxantel pamoate with a CR of $68.5 \%$ [59.6-77.4\%] [33•] followed by combinations of 
Table 2 Summary of cure rates and egg reduction rate of combination therapies in trichuriasis

\begin{tabular}{lll}
\hline & Cure rate in \%[95\% CI $]$ & Egg reduction rate in \%[95\% CI $]$ \\
\hline Albendazole+ivermectin & $27.5[19.0-36.0]^{\mathrm{a}}[33 \bullet]$ & $94.5[91.7-96.3]^{\mathrm{a}}[33 \bullet]$ \\
Albendazole+oxantel pamoate & $68.5[59.6-77.4]^{\mathrm{a}}[33 \bullet] ;$ & $99.2[98.7-99.6]^{\mathrm{a}}[33 \bullet] ;$ \\
& $31.2[22.5-40]^{\mathrm{b}}[26 \bullet]$ & $96.0[93.5-97.6]^{\mathrm{b}}[26 \bullet]$ \\
Albendazole+mebendazole & $8.4[3.1-13.8]^{\mathrm{a}}[33 \bullet] ;$ & $51.6[35.0-65.3]^{\mathrm{a}}[33 \bullet] ;$ \\
Albendazole+mebendazole/ & $54.2[43.6-64.8]^{\mathrm{a}}[46 \bullet]$ & $94.3^{\mathrm{a}}[46 \bullet]^{\mathrm{d}}$ \\
albendazole+mebendazole & $56.5[46.4-66.7]^{\mathrm{c}}[46 \bullet]$ & $95.9^{\mathrm{c}}[46 \bullet]^{\mathrm{d}}$ \\
Mebendazole+mebendazole & $41.9[31.9-52.0]^{\mathrm{c}}[46 \bullet]$ & $94.3^{\mathrm{c}}[46 \bullet]^{\mathrm{d}}$ \\
Albendazole+nitazoxanide & $16.0[9.7-22.4]^{\mathrm{a}}[29 \bullet]$ & $54.9[37.7-67.9]^{\mathrm{a}}[29 \bullet]$ \\
\hline
\end{tabular}

${ }^{a}$ Drugs were given once at the same time

${ }^{\mathrm{b}}$ Drugs were given on two consecutive days

${ }^{\mathrm{c}}$ Drugs were given $8 \mathrm{~h}$ apart on the same day

${ }^{\mathrm{d}} 95 \%$ confidence intervals are missing in this study for ERR two albendazole doses of $400 \mathrm{mg}$ plus two mebendazole doses of $500 \mathrm{mg}$ administered $8 \mathrm{~h}$ apart, which achieved a CR of $56 \%$ [46.4-66.7\%] [46•], and albendazole plus ivermectin which achieved a CR of $27.5 \%$ [19.0-36.0\%]. The poorest CR was achieved with a combination of albendazole plus nitazoxanide (16.0\% [9.7\%-22.4\%]) [29•] (Table 2). The same trend also applies to ERR, where the highest reduction was seen with albendazole plus oxantel pamoate $(99.2 \%$ [98.7-99.6\%]) [33•]. The lowest ERR was observed for a combination of albendazole and mebendazole (51.6\% [35.0-65.3\%]) [33•] (see Table 2). In summary, repeated doses as well as combination therapy yield better efficacy in comparison to a single-dose regimen.

\section{Resistance of T. trichiura to Anthelmintic Drugs}

Benzimidazole drugs have been widely used against STHs for decades in the treatment of individual patients and in mass drug administration programmes. Selection pressure caused by these drugs raised the question of potentially emerging resistant populations of STHs. Drug resistance may explain the poor cure rates in infection with $T$. trichiura using a benzimidazole therapy alone or in combination with other drugs. Benzimidazole drugs are broad spectrum anthelmintics that bind to tubulin, causing interference with tubulin polymerization and destabilization of microtubules [48]. In veterinary studies, it has been observed that the substitution of a single amino acid (from phenylalanine (Phe, TTC) to tyrosine (Tyr, TAC) in the $\beta$-tubulin at position 200 may be associated with benzimidazole resistance) [49]. In cattle trichuriasis, several single nucleotide polymorphisms (SNPs) in the $\beta$-tubulin isotype 1 gene of various nematodes correlate with resistance to benzimidazoles [50]. In humans, occurrence of TAC SNP at codon 200 in $T$. trichiura is suspected to be potentially associated with resistance to benzimidazoles [51•]. Hence, these findings may potentially explain the poor cure rates in the treatment of $T$. trichiura infestation using benzimidazole drugs, if further substantiated. However, more data, prospective studies and close monitoring of cure rates and genetic markers of drugs resistance are necessary $[11 \cdot]$.

\section{Conclusion}

Human T. trichiura is highly endemic in tropical regions, and most current therapeutic regimens require either prolonged treatment courses or have unsatisfactory cure rates. These poor outcomes may potentially be explained by the presence of genetic markers including a SNP in the beta-tubulin gene at codon 200 which was reported to be associated with benzimidazole drug resistance. Further clinical research aiming to improve cure rates and shortening treatment regimens is therefore warranted as well as studies adding to our knowledge of genetic markers for clinical drug resistance.

Acknowledgments AAA is supported by the EU-funded project Health-F3-2013-602843 FP7 GA HOOKVAC.

\section{Compliance with Ethics Guidelines}

Conflict of Interest The authors declare that they have no competing interests.

Human and Animal Rights and Informed Consent This article does not contain any studies with human or animal subjects performed by any of the authors. 


\section{References}

Papers of particular interest, published recently, have been highlighted as:

- Of importance

1. Brooker S, Clements AC, Bundy DA. Global epidemiology, ecology and control of soil-transmitted helminth infections. Adv Parasitol. 2006;62:221-61.

2. Hotez PJ, Kamath A. Neglected tropical diseases in sub-saharan Africa: review of their prevalence, distribution, and disease burden. PLoS Negl Trop Dis. 2009;3:e412.

3. Hotez PJ. A constitutional amendment for deworming. PLoS Negl Trop Dis. 2009;3:e454.

4. Albonico M, Allen H, Chitsulo L, Engels D, Gabrielli AF, Savioli L. Controlling soil-transmitted helminthiasis in pre-school-age children through preventive chemotherapy. PLoS Negl Trop Dis. 2008;2:e126.

5. Galvani AP. Age-dependent epidemiological patterns and strain diversity in helminth parasites. J Parasitol. 2005;91:24-30.

6. Kattula D, Sarkar R, Rao Ajjampur SS, Minz S, Levecke B, Muliyil $\mathrm{J}$, et al. Prevalence \& risk factors for soil transmitted helminth infection among school children in south India. Indian J Med Res. 2014;139:76-82.

7. Davis SM, Worrell CM, Wiegand RE, Odero KO, Suchdev PS, Ruth LJ, et al. Soil-transmitted helminths in pre-school-aged and school-aged children in an urban slum: a cross-sectional study of prevalence, distribution, and associated exposures. Am J Trop Med Hyg. 2014;91:1002-10.

8. Adegnika AA, Agnandji ST, Chai SK, Ramharter M, Breitling L, Kendjo E, et al. Increased prevalence of intestinal helminth infection during pregnancy in a Sub-Saharan African community. Wien Klin Wochenschr. 2007;119:712-6.

9. Campbell SJ, Savage GB, Gray DJ, Atkinson JA, Soares Magalhaes RJ, Nery SV, et al. Water, Sanitation, and Hygiene (WASH): a critical component for sustainable soil-transmitted helminth and schistosomiasis control. PLoS Negl Trop Dis. 2014;8: e2651.

10. World Health Organization, Communicable DC, Dept.of Communicable Disease Prevention and Control. Report of the WHO informal consultation on monitoring of drug efficacy in the control of schistosomiasis and intestinal nematodes, Geneva, 8-10 July 1998. Geneva: World Health Organization; 1999.

11. Diawara A, Schwenkenbecher JM, Kaplan RM, Prichard RK. Molecular and biological diagnostic tests for monitoring benzimidazole resistance in human soil-transmitted helminths. Am J Trop Med Hyg. 2013;88:1052-61. This paper described molecular assays to detect putative resistance markers on STH.

12. Chan MS, Medley GF, Jamison D, Bundy DA. The evaluation of potential global morbidity attributable to intestinal nematode infections. Parasitology. 1994;109(Pt 3):373-87.

13. Hotez PJ, Molyneux DH, Fenwick A, Kumaresan J, Sachs SE, Sachs JD, et al. Control of neglected tropical diseases. N Engl J Med. 2007;357:1018-27.

14. Brooker S, Akhwale W, Pullan R, Estambale B, Clarke SE, Snow RW, et al. Epidemiology of plasmodium-helminth co-infection in Africa: populations at risk, potential impact on anemia, and prospects for combining control. Am J Trop Med Hyg. 2007;77:88-98.

15. Dreyfuss ML, Stoltzfus RJ, Shrestha JB, Pradhan EK, LeClerq SC, Khatry SK, et al. Hookworms, malaria and vitamin A deficiency contribute to anemia and iron deficiency among pregnant women in the plains of Nepal. J Nutr. 2000;130:2527-36.
16. Katona P, Katona-Apte J. The interaction between nutrition and infection. Clin Infect Dis. 2008;46:1582-8.

17. Hesham MS, Edariah AB, Norhayati M. Intestinal parasitic infections and micronutrient deficiency: a review. Med J Malaysia. 2004;59:284-93.

18. Mireku MO, Boivin MJ, Davidson LL, Ouedraogo S, Koura GK, Alao MJ, et al. Impact of helminth infection during pregnancy on cognitive and motor functions of one-year-old children. PLoS Negl Trop Dis. 2015;9:e0003463.

19. Elliott AM, Namujju PB, Mawa PA, Quigley MA, Nampijja M, Nkurunziza PM, et al. A randomised controlled trial of the effects of albendazole in pregnancy on maternal responses to mycobacterial antigens and infant responses to Bacille Calmette-Guerin (BCG) immunisation [ISRCTN32849447]. BMC Infect Dis. 2005;5:115.

20. van Riet E, Adegnika AA, Retra K, Vieira R, Tielens AG, Lell B, et al. Cellular and humoral responses to influenza in gabonese children living in rural and semi-urban areas. J Infect Dis. 2007;196: 1671-8.

21. van Riet E, Retra K, Adegnika AA, der Zijde CM J-v, Uh HW, Lell $\mathrm{B}$, et al. Cellular and humoral responses to tetanus vaccination in Gabonese children. Vaccine. 2008;26:3690-5.

22. Esen M, Mordmuller B, de Salazar PM, Adegnika AA, Agnandji ST, Schaumburg F, et al. Reduced antibody responses against Plasmodium falciparum vaccine candidate antigens in the presence of Trichuris trichiura. Vaccine. 2012;30:7621-4.

23. World Health Organization. Soil-transmitted helminthiases eliminating as public health problem soil-transmitted helminthiases in children : progress report 2001-2010 and strategic plan 20112020. Geneva: World Health Organization; 2012.

24. Horton J. Global anthelmintic chemotherapy programs: learning from history. Trends Parasitol. 2003;19:405-9.

25. Adegnika AA, Zinsou JF, Issifou S, Ateba-Ngoa U, Kassa RF, Feugap EN, et al. Randomized, controlled, assessor-blind clinical trial to assess the efficacy of single- versus repeated-dose albendazole to treat ascaris lumbricoides, trichuris trichiura, and hookworm infection. Antimicrob Agents Chemother. 2014;58: 2535-40. This randomized controlled clinical trial assesses single dose versus repeated-dose albendazole treatment for STH including T. trichirua. Three day albendazol treatment was significantly more efficacious than single dose treatment.

26. Speich B, Ame SM, Ali SM, Alles R, Huwyler J, Hattendorf J, et al. Oxantel pamoate-albendazole for Trichuris trichiura infection. $\mathrm{N}$ Engl J Med. 2014;370:610-20. This randomized controlled clinical trial assessed the efficacy of oxantel pamoat-albendazol combination agains $T$. trichiura compared to mebendazol therapy. Cure rate was higher although still disappointingly low at $31.2 \%$.

27. Namwanje H, Kabatereine N, Olsen A. A randomised controlled clinical trial on the safety of co-administration of albendazole, ivermectin and praziquantel in infected schoolchildren in Uganda. Trans RSoc Trop Med Hyg. 2011;105:181-8.

28. Samuel F, Degarege A, Erko B. Efficacy and side effects of albendazole currently in use against Ascaris, Trichuris and hookworm among school children in Wondo Genet, southern Ethiopia. Parasitol Int. 2014;63:450-5. Single dose albendazole treatment of $T$. trichiura was associated with $31 \%$ cure rate in ths clincial trial conducted in Ethiopia.

29. Speich B, Ame SM, Ali SM, Alles R, Hattendorf J, Utzinger J, et al. Efficacy and safety of nitazoxanide, albendazole, and nitazoxanidealbendazole against Trichuris trichiura infection: a randomized controlled trial. PLoS Negl Trop Dis. 2012;6:e1685. Nitazoxanide was assessed in comparison with albendazole and nitazoxanidealbendazole combination in a randomized controlled clinical trial. Nitazoxanide proved to be ineffective against $T$. trichiura with a cure rate of only $7 \%$. 
30. Yap P, Du ZW, Wu FW, Jiang JY, Chen R, Zhou XN, et al. Rapid reinfection with soil-transmitted helminths after triple-dose albendazole treatment of school-aged children in Yunnan, People's Republic of China. Am J Trop Med Hyg. 2013;89:2331. Three day treatment of $\mathbf{T}$. trichiura with albendazole led to $20 \%$ cure rate in this Chinese population. Re-infection with T. trichiura was slower than for other STH.

31. Geary TG. Are new anthelmintics needed to eliminate human helminthiases? Curr Opin Infect Dis. 2012;25:709-17.

32. Mpairwe H, Tweyongyere R, Elliott A. Pregnancy and helminth infections. Parasite Immunol. 2014;36:328-37.

33. Speich B, Ali SM, Ame SM, Bogoch II, Alles R, Huwyler J, et al. Efficacy and safety of albendazole plus ivermectin, albendazole plus mebendazole, albendazole plus oxantel pamoate, and mebendazole alone against Trichuris trichiura and concomitant soil-transmitted helminth infections: a four-arm, randomised controlled trial. Lancet Infect Dis. 2015;15:277-84. A four arm headto -head comparison of combinations therapies for T. trichiura demonstrated that albendazole in combination with oxantel pamoate leads to highest cure rates.

34. Adams EJ, Stephenson LS, Latham MC, Kinoti SN. Physical activity and growth of Kenyan school children with hookworm, Trichuris trichiura and Ascaris lumbricoides infections are improved after treatment with albendazole. J Nutr. 1994;124:1199-206.

35. Stephenson LS, Latham MC, Adams EJ, Kinoti SN, Pertet A. Physical fitness, growth and appetite of Kenyan school boys with hookworm, Trichuris trichiura and Ascaris lumbricoides infections are improved four months after a single dose of albendazole. J Nutr. 1993;123:1036-46.

36. Stephenson LS, Latham MC, Adams EJ, Kinoti SN, Pertet A. Weight gain of Kenyan school children infected with hookworm, Trichuris trichiura and Ascaris lumbricoides is improved following once- or twice-yearly treatment with albendazole. J Nutr. 1993;123: 656-65.

37. Elliott AM, Ndibazza J, Mpairwe H, Muhangi L, Webb EL, Kizito $\mathrm{D}$, et al. Treatment with anthelminthics during pregnancy: what gains and what risks for the mother and child? Parasitology. 2011;138:1499-507.

38. Ndibazza J, Mpairwe H, Webb EL, Mawa PA, Nampijja M, Muhangi $\mathrm{L}$, et al. Impact of anthelminthic treatment in pregnancy and childhood on immunisations, infections and eczema in childhood: a randomised controlled trial. PLoS One. 2012;7:e50325.

39. Mpairwe H, Webb EL, Muhangi L, Ndibazza J, Akishule D, Nampijja M, et al. Anthelminthic treatment during pregnancy is associated with increased risk of infantile eczema: randomisedcontrolled trial results. Pediatr Allergy Immunol. 2011;22:305-12.

40. Webb EL, Mawa PA, Ndibazza J, Kizito D, Namatovu A, Kyosiimire-Lugemwa J, et al. Effect of single-dose anthelmintic treatment during pregnancy on an infant's response to immunisation and on susceptibility to infectious diseases in infancy: a randomised, double-blind, placebo-controlled trial. Lancet. 2011;377:52-62.
41. Atukorala TM, de Silva LD, Dechering WH, Dassenaeike TS, Perera RS. Evaluation of effectiveness of iron-folate supplementation and anthelminthic therapy against anemia in pregnancy-a study in the plantation sector of Sri Lanka. Am J Clin Nutr. 1994;60:286-92.

42. de Silva NR, Sirisena JL, Gunasekera DP, Ismail MM, de Silva HJ. Effect of mebendazole therapy during pregnancy on birth outcome. Lancet. 1999;353:1145-9.

43. Torlesse H, Hodges M. Albendazole therapy and reduced decline in haemoglobin concentration during pregnancy (Sierra Leone). Trans RSoc Trop Med Hyg. 2001;95:195-201.

44. Torlesse H, Hodges M. Anthelminthic treatment and haemoglobin concentrations during pregnancy. Lancet. 2000;356:1083.

45. Levecke B, Montresor A, Albonico M, Ame SM, Behnke JM, Bethony JM, et al. Assessment of anthelmintic efficacy of mebendazole in school children in six countries where soiltransmitted helminths are endemic. PLoS Negl Trop Dis. 2014;8: e3204. This analysis of multiple trials assessing mebendazole and albendazole for egg reduction in $T$. trichiura demonstrated equal egg reduction rates for mebendazole and albendazole.

46. Namwanje H, Kabatereine NB, Olsen A. Efficacy of single and double doses of albendazole and mebendazole alone and in combination in the treatment of Trichuris trichiura in school-age children in Uganda. Trans RSoc Trop Med Hyg. 2011;105:586-90. This clinical trial evaluated the impact of doubling the dose of benzimidazole therapy for $T$. trichiura. The main finding is an increased efficacy of double dose treatment and of combination of mebendazole with albendazole

47. Steinmann P, Utzinger J, Du ZW, Jiang JY, Chen JX, Hattendorf J, et al. Efficacy of single-dose and triple-dose albendazole and mebendazole against soil-transmitted helminths and Taenia spp.: a randomized controlled trial. PLoS One. 2011;6:e25003. This study assessed the efficacy of benzimidazole therapy against STH when given as single dose or triple dose regimens. Triple dose regimens were more efficacious against $T$. trichiura.

48. Lacey E. Mode of action of benzimidazoles. Parasitol Today. 1990;6:112-5.

49. Wolstenholme AJ, Fairweather I, Prichard R, von SamsonHimmelstjerna G, Sangster NC. Drug resistance in veterinary helminths. Trends Parasitol. 2004;20:469-76.

50. Demeler J, Kruger N, Krucken J, von der Heyden VC, Ramunke S, Kuttler U, et al. Phylogenetic characterization of beta-tubulins and development of pyrosequencing assays for benzimidazole resistance in cattle nematodes. PLoS One. 2013;8:e70212.

51. Diawara A, Drake LJ, Suswillo RR, Kihara J, Bundy DA, Scott ME, et al. Assays to detect beta-tubulin codon 200 polymorphism in Trichuris trichiura and Ascaris lumbricoides. PLoS Negl Trop Dis. 2009;3:e397. This study describes pyroseuqencing assays for the detection of molecular markers of benzimidazole resistance. Assays were employed in treatment naive populationas and in populations with intensive mass treatments and the authors discuss the implications of detected resistance markers. 